\title{
ULRIKE DEMSKE
}

\section{Das Mercurius-Projekt: Eine Baumbank für das Frühneuhochdeutsche}

\begin{abstract}
Wer sich mit grammatischen Phänomenen historischer Sprachstufen beschäftigt, kann seine empirischen Daten bekanntermaßen nicht auf der Grundlage von Sprecherurteilen gewinnen, sondern muss zunächst Korpusrecherchen betreiben. Die Größe des auszuwählenden Korpus ist sehr stark phänomenabhängig: So reicht es im Bereich der Syntax in der Regel nicht aus, kleinere Textausschnitte aus verschiedenen Textquellen zu einem Korpus zusammenzufügen, vielmehr müssen vollständige Texte nicht nur nach raum-zeitlichen Koordinaten, sondern auch textsortenabhängig ausgewählt werden, um ein repräsentatives Korpus für eine spezifische Sprachstufe zu erstellen. Da eine manuelle Sichtung dieser doch recht großen Korpora sich sehr zeitaufwändig gestaltet, bietet sich gerade im Bereich der historischen Syntax der Einsatz von syntaktisch annotierten, digitalen Korpora an. Im folgenden Beitrag wird der Aufbau einer solchen Baumbank für das Frühneuhochdeutsche einschließlich der verfügbaren Recherchemöglichkeiten vorgestellt.
\end{abstract}

\section{Einleitung}

Die rasch zunehmende Verfügbarkeit digitalisierter Texte prägt seit einer Reihe von Jahren maßgeblich den methodischen Zugang in ganz unterschiedlichen Wissenschaftsdisziplinen, darunter auch den zu sprachwissenschaftlichen Fragestellungen. Die sprachwissenschaftliche Nutzung von Korpora erstreckt sich vor allem auf lexikologische Unternehmungen wie die Erstellung von Wörterbüchern oder von Kollokationslisten, wie sie beispielsweise durch die am Institut für Deutsche Sprache entwickelten Suchwerkzeuge von ,Cosmas' und den dort verfügbaren Korpora ermöglicht werden. Erst in jüngerer Zeit erlauben Entwicklungen in der Computerlinguistik eine weiter gehende linguistische Interpretation größerer Korpora und damit die elektronische Suche nicht mehr allein nach Wörtern oder Wortketten, sondern nach syntaktischen Strukturen. Es handelt sich hier um eine Form der Empirie, die von der primär gegenwartssprachlich ausgerichteten Syntaxforschung erst in den letzten Jahren wirklich ernst genommen wird. Diachron orientierte Forschung dagegen ist durch das Fehlen negativer Evidenz per se korpuslinguistisch fundiert, da für jede historisch ausgerichtete Fragestellung ein der Fragestellung angemessenes Textkorpus zusammengestellt und ausgewertet werden muss. So unterstreicht Ebert (1986, S. 23), dass jede Unter- 
suchung von Sprachwandelphänomenen auf einem repräsentativen Korpus beruhen muss, das es erlaubt, den Einfluss von zeitlichen, geographischen, sozialen und stilistischen Faktoren auf den fraglichen Wandel zuverlässig einschätzen zu können. Es liegt auf der Hand, dass sich diese Forderung nach Repräsentativität ${ }^{1}$ umso schwieriger erfüllen lässt, desto geringer die Auftretensdichte des zu untersuchenden Phänomens ist. Ein Beispiel möge dies veranschaulichen: Um die Flexionsmorphologie des Frühneuhochdeutschen in ihren wesentlichen Grundzügen zu beschreiben, war es nach Solms/Wegera (1998) ausreichend, unter Berücksichtigung von zeitlichen, geographischen und stilistischen Faktoren Texte nicht zwangsläufig in ihrer Gesamtheit auf die relevanten sprachlichen Eigenschaften hin auszuwerten, vielmehr konnte diese Auswertung auf Textausschnitte von etwa 12.000 laufenden Wortformen beschränkt werden. Aufgrund einer deutlich geringeren Belegdichte kann die Untersuchung von syntaktischem Wandel im Unterschied dazu jedoch ausschließlich von Volltexten ausgehen. Und hier erlaubt die zunehmende Digitalisierung historischer Texte die Sichtung weit größerer Datenmengen als es bislang durch die rein manuelle Auswertung möglich war. Solange die vorliegenden Korpora aber reine Textkorpora ohne Anreicherung durch syntaktische Informationen sind, ist für die historische Syntax nicht viel gewonnen. Eine Auswertung solcher Korpora für syntaktische Fragestellungen würde nur dann einen Vorteil gegenüber ausschließlich in Papierform vorliegenden Texten erbringen, wenn es um syntaktische Phänomene ginge, die zwingend einen sichtbaren Reflex in der Morphologie haben (etwa der Kasusmorphologie bei der Suche nach Argumentstrukturen). Das gilt jedoch nur für eine äußerst geringe Zahl von syntaktischen Phänomenen. Für die Mehrzahl setzt die Suche nach syntaktischen Mustern voraus, dass die digitalisierten Texte mit syntaktischen Informationen angereichert sind, wie das im Rahmen von sog. Baumbanken geleistet wird. ${ }^{2}$ Für das Gegenwartsdeutsche sind mit ,Negra' und ,Tiger' solche Baumbanken vorgelegt worden (Skut et al. 1998, Brants et al. 1999, Brants et al. 2002). Für historische Sprachstufen des Deutschen bestehen bislang keine vergleichbaren Korpora. Hier soll das seit 2003 an der Universität des Saarlandes etablierte MercuriusProjekt Abhilfe schaffen: Ziel ist der Aufbau einer Baumbank auf der Grundlage von Zeitungstexten der Frühen Neuzeit. Da Baumbanken Informationen

1 Wie Biber (1993) ausführt, ist die Größe eines Korpus nur mittelbar ein Faktor für seine Repräsentativität. Repräsentativität bezieht sich vielmehr auf das Maß, zu dem ein Korpus den vollen Umfang von Varianten in einer Sprachgemeinschaft einschließt. Und diese Varianten können außer- oder innersprachlich bedingt sein. Vor der Zusammenstellung eines Korpus müssen deshalb Situationsparameter und linguistische Merkmale identifiziert werden, um dem Ziel der Repräsentativität eines Korpus möglichst nahe zu kommen; zum Aufbau strukturierter Korpora s. auch Wegera (2000). Von Lüdeling (in diesem Band) wird die Auffassung vertreten, dass von Repräsentativität nur bei Kenntnis der Grundgesamtheit gesprochen werden darf.

2 Zur Einführung s. Lezius (2001). 
auf unterschiedlichen Ebenen der syntaktischen Analyse enthalten, sind sie im Unterschied zu anderen Ergebnisdatenbanken nicht phänomenorientiert, sondern lassen sich mittels geeigneter Abfragen nach immer neuen syntaktischen Mustern durchsuchen.

Im vorliegenden Beitrag werde ich über den Aufbau der MercuriusBaumbank berichten sowie auf einige Probleme eingehen, die sich bei der Anreicherung historischer Texte mit syntaktischen Informationen stellen. Abschließend werden beispielhaft die zur Verfügung stehenden Recherchemöglichkeiten vorgestellt.

\section{Die Mercurius-Baumbank}

\subsection{Textgrundlage}

Erster und namengebender Baustein der Baumbank ist ein Zeitungstext aus dem Jahr 1667, der wöchentlich in Hamburg erschienene, Nordische Mercurius', herausgegeben von Georg Greflinger. ${ }^{3}$ Folgende Gründe sprechen dafür, den Aufbau eines syntaktisch annotierten Korpus mit einem Text aus der Frühen Neuzeit zu beginnen: Ein solcher Text bietet sich für eine Erprobung der Annotationswerkzeuge schon deshalb an, weil wir es hier mit der neuhochdeutschen Standardsprache in statu nascendi zu tun haben, die zunächst eine ausschließlich geschriebene Varietät des Deutschen mit entsprechend komplexen syntaktischen Strukturen darstellt (Glaser 2003, von Polenz 1994). Die aus der Literatur hinreichend bekannte Komplexität von Satzstrukturen im Frühneuhochdeutschen (Admoni 1980, Ebert 1986) mag folgendes Beispiel aus dem Mercurius illustrieren, in dem sechs Teilsätze in einem Satzgefüge verschachtelt sind. Satzgefüge wie unter (1) stellen im Mercurius eher die Regel als die Ausnahme dar.

(1) Von dem Ober-Ungar. General sind dieser Tagen Briefe eingekommen/wie daß die Türcken in Siebenbürgen 2. grosse Machten/ als eine bey dem Schloß Jenona/die andere aber bey der festen Insul Jula versamleten/und daß sie muthmassentlich des Vorhabens seyen/ entweder den Fürsten Abaff// weiln er die der Pforten placidirte Contributiones nicht abführen kan/ absetzen/oder aber etliche Berg-Städte/wo der beste Siebenbürgische Wein wächst/ in der Türcken devotion zu bringen/ (M 8.8) ${ }^{4}$

Es wird deshalb angenommen, dass bei der syntaktischen Anreicherung der Texte sehr schnell die möglichen Grenzen eines Annotationswerkzeugs zu Tage treten und an entsprechenden Modifikationen des Werkzeugs gearbeitet werden kann, die dann für alle Stufen der deutschen Sprachgeschichte nutzbar sind. Gleichzeitig muss berücksichtigt werden, dass die bestehenden Werkzeuge für das Gegenwartsdeutsche entwickelt worden sind. Aufgrund dessen

3 Für Details s. Gieseler/Kühnle-Xemaire (1995) sowie Böning (2005).

4 Angegeben ist jeweils die Seiten- und Zeilenzahl des Beleganfangs. 
empfiehlt es sich aus rein technischen Gründen, mit der Annotation von Texten zu beginnen, die in ihrem Lexikon und ihrem grammatischen System noch eine starke Übereinstimmung mit dem Gegenwartsdeutschen aufweisen. Das wird im nächsten Abschnitt deutlich werden, wenn die Annotationsschritte im Einzelnen vorgestellt und erläutert werden.

\subsection{Annotation}

Vor der syntaktischen Annotation der Texte steht ihre Digitalisierung: Im Fall des Mercurius wurde diese Digitalisierung auf der Grundlage des Jahrgangs 1667 vorgenommen. ${ }^{5}$ Einen typischen Ausschnitt aus der auf Korrespondenzen basierenden Ausgabe zeigt Abb. 1:

\section{Frandret(f).}

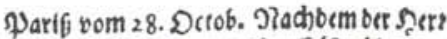
Bifthoff von Whinfer mit Ëur(s dan úber oo Eoabjutourfahaffe von MRüfer m Differentien comme/fo baben Ë is ter, betibre Brefanbern blefer

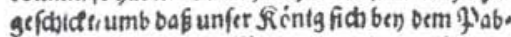
fec wegenibrer Interefle interponiren módate. Der Graf von Pútfenberg Wotfchoff it Dla negotiiret bor unfern Söonig bev bell Deut(d)ent

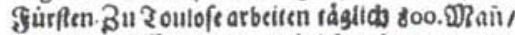
umbeinen tieffen Şaven Dafelbf bu baben I uno groffe Soduffe su legen. Bu Earcaffone motro an groffen $23 \mathrm{ken}$ Wh inen/fo juing ft entberdet morben/

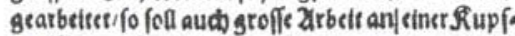
fer WRine gefacheben I retide man beybejublefes Retidics groffen Zotibeil cractett. Die recru-

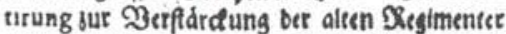
gefdeben mit ungemeinem Eufer. Ltmb Das S fft

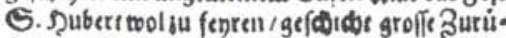

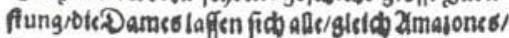

\section{Franckreich.}

Pariß vom 28. Octob. Nachdem der Herr
Bischoff von Münster mit Cur Colln uber der
Coadjutourschafft von Munster in Differentien
kommt/ so haben Sie beyde ihre Gesandten hieher
geschickt/ umb daß unser König sich bey dem Pab=
ste wegen ihrer Interesse interponiren mochte.
Der Graf von Furstenberg Bischoff zu Mez
negotiiret vor unsern Konig bey den Deutschen
Fursten. Zu Toulose arbeiten täglich 800 . Mañ/
umb einen tieffen Haven daselbst zu haben/ und
grosse Schiffe zu legen. Zu Carcassone wird an
grossen Bley Minen/ so jungst entdecket worden/
gearbeitet/ so soll auch grosse Arbeit an: einer Kupf=
fer Mine geschehen/ welche man beyde zu dieses
Reiches grossen Vortheil erachtet. Die recru=
tirung zur Verstarckung der alten Regimenter
geschehen mit ungemeinem Eufer. Umb das Fest
S. Hubert wol zu feyren/ geschicht grosse Zuru=
stung/ die Dames lassen sich alle/ gleich Amazones/

Abb. 1: Digitalisierung von Textquellen

Der erste Schritt auf dem Weg zu einem syntaktisch annotierten Korpus umfasst die Zerlegung des Textes in Satzeinheiten, d. h. seine Tokenisierung. Bereits bei dieser Arbeitsaufgabe manifestiert sich ein entscheidender Unterschied zwischen den im Rahmen der Negra- und Tigerprojekte bearbeiteten Texten aus der Gegenwart einerseits und dem Text aus der Frühen Neuzeit andererseits: Während für die Texte aus dem heutigen Deutsch im Wesentlichen davon ausgegangen werden kann, dass Satzeinheiten durch Punkte abzugrenzen sind, markieren Satzzeichen im Frühneuhochdeutschen Satzgrenzen nur sehr unzuverlässig (Stolt 1990). Dieses Problem möchte ich an

5 Die Digitalisierung des Nordischen Mercurius war Teil des DFG-Projekts ,Entstehung und Entwicklung der Zeitungssprache im 17. Jahrhundert', das in den Jahren 1987 bis 1992 unter der Leitung von Prof. Erich Straßner und Prof. Gerd Fritz an der Universität Tübingen durchgeführt wurde. 
einem Textausschnitt illustrieren: Der folgende Ausschnitt enthält insgesamt drei Satzgefüge, von denen allein der Umfang des letzten Satzgefüges unstrittig ist. Der Textteil vor dem Absatz ist durch Punkte in zwei Satzeinheiten gegliedert: Der Beginn des ersten Satzes fällt mit dem Beginn des Textausschnitts zusammen $(=E s)$, der zweite Satz beginnt laut Interpunktion und Majuskel mit dem Pronominaladverb worüber. Inhaltlich bilden beide Sätze eine Einheit. Unter syntaktischen Gesichtspunkten wäre eine Satzgrenze allerdings eher vor dem ebenfalls hervorgehobenen Mons(ieur) zu setzen, da hier mit einem V2-Satz eine syntaktisch unabhängige Einheit beginnt, wohingegen das mit einer Majuskel beginnende worüber einen V-letzt-Satz einleitet. Im Gegenwartsdeutschen sind diese weiterführenden Nebensätze abhängige Satzteile größerer Satzgefüge.

(2) Es sind viel/ welche vermeynen/ daß man allhier nicht die beste Opinion gegen Franckreich habe/ aus Ursachen/weil sie in der Levante die unsrige nicht zum besten meynen sollen Mons. d'Almeras soll damahls/als die Veränderung in Portugall vorgegangen/mit vielen Frantzösischen Schiffen auff der Revier von Lisabona gewesen seyn. Worüber auch seltzame Gedancken entstehen.

Heute hat die Königl. Societät die Uberbringung des Blutes aus einem Schafe in einen Menschen experimentirt, welches wol succedirte vor die quantität von 12 à 14 Untzen. (M 813.27)

Die Schlussfolgerung, die meines Erachtens aus Textbeispielen wie (2) zu ziehen ist, lautet, dass die Tokenisierung historischer Texte anders als im Gegenwartsdeutschen nicht automatisch zu leisten ist, sondern entweder manuell oder halbautomatisch zu erfolgen hat. Auf jeden Fall müssen vor der Tokenisierung die einschlägigen (syntaktischen) Kriterien für Satzwertigkeit genauestens festgeschrieben werden (Demske-Neumann 1990, Demske et al. 2004).

Die nächsten Arbeitsschritte beinhalten die Anreicherung der Satztokens mit syntaktischen Informationen; im Einzelnen sind das die Wortartkodierung ${ }^{6}$ sowie die Auszeichnung von Phrasentypen (runde Umrahmung) und Funktionen von Phrasen (eckige Umrahmung). Wie das im Detail aussieht, wird im Folgenden am Beispiel der Analyse eines Teilsatzes aus dem Textausschnitt (2) illustriert.

Wie die Abbildung zeigt, sind die syntaktischen Strukturen sehr flach und einfach aufgebaut: Argumente und Modifikatoren werden nicht positionell, sondern über eine Funktionsbezeichnung unterschieden $(\mathrm{OA}=$ Akkusativobjekt, $\mathrm{MO}=$ Modifikator) . Das erklärte Ziel besteht hier in der Vermeidung von Ambiguitäten beim Aufbau von Strukturen (Brants et al. 2002). Unbestreitbarer Vorteil des verwendeten Annotationsschemas ist die Möglichkeit, Abhängigkeitsverhältnisse zwischen Konstituenten auch durch sich kreuzende Äste im Baumdiagramm darzustellen. Damit kann dem häufigen Auftreten

6 Zur Kodierung der Wortarten verwendet ,Annotate' das Stuttgart/Tübinger Tagset (STTS) mit Modifikationen, s. Smith/ Eisenberg (2000). 


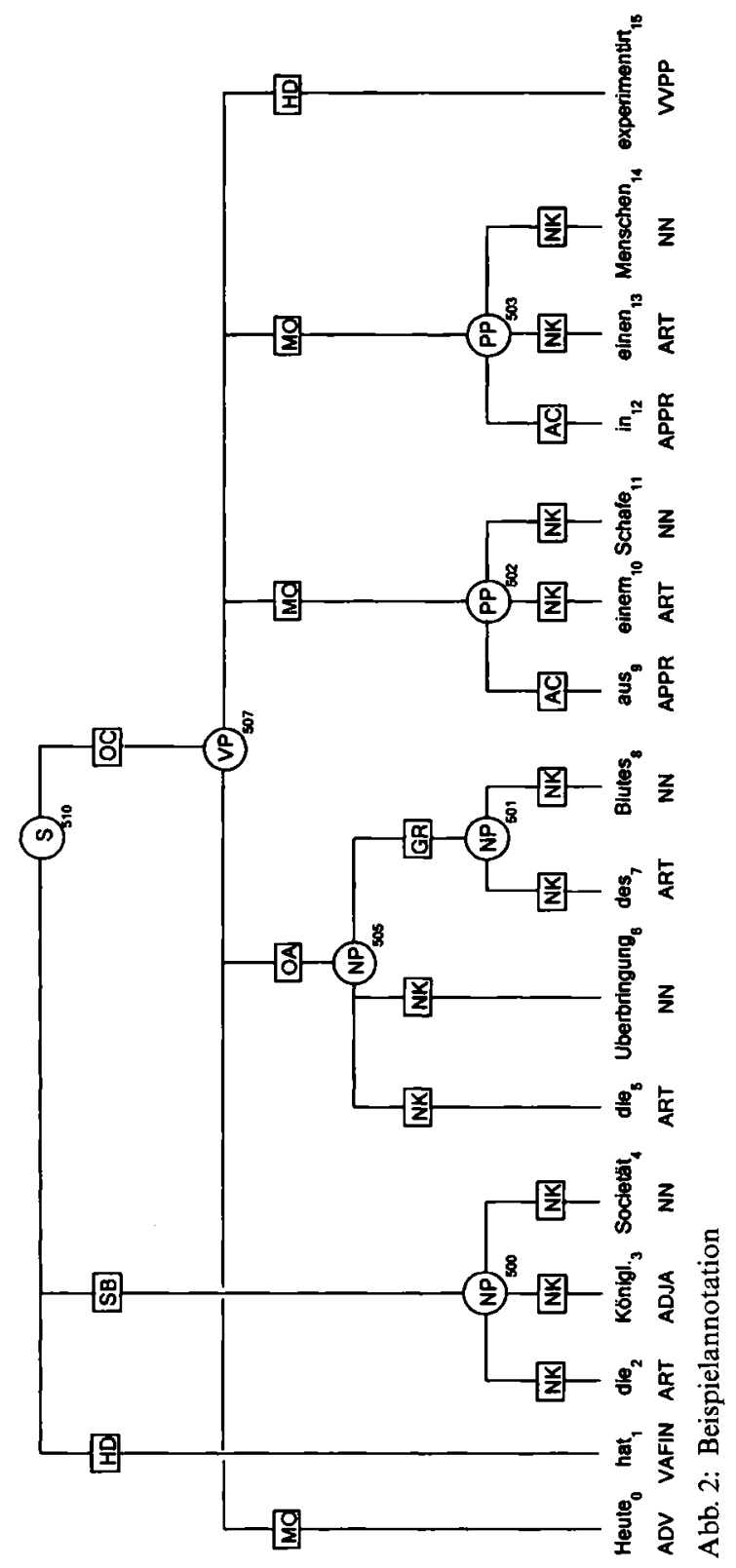


von diskontinuierlichen Konstituenten im Frühneuhochdeutschen Rechnung getragen werden, wie hier am Beispiel eines extraponierten Relativsatzes gezeigt wird.

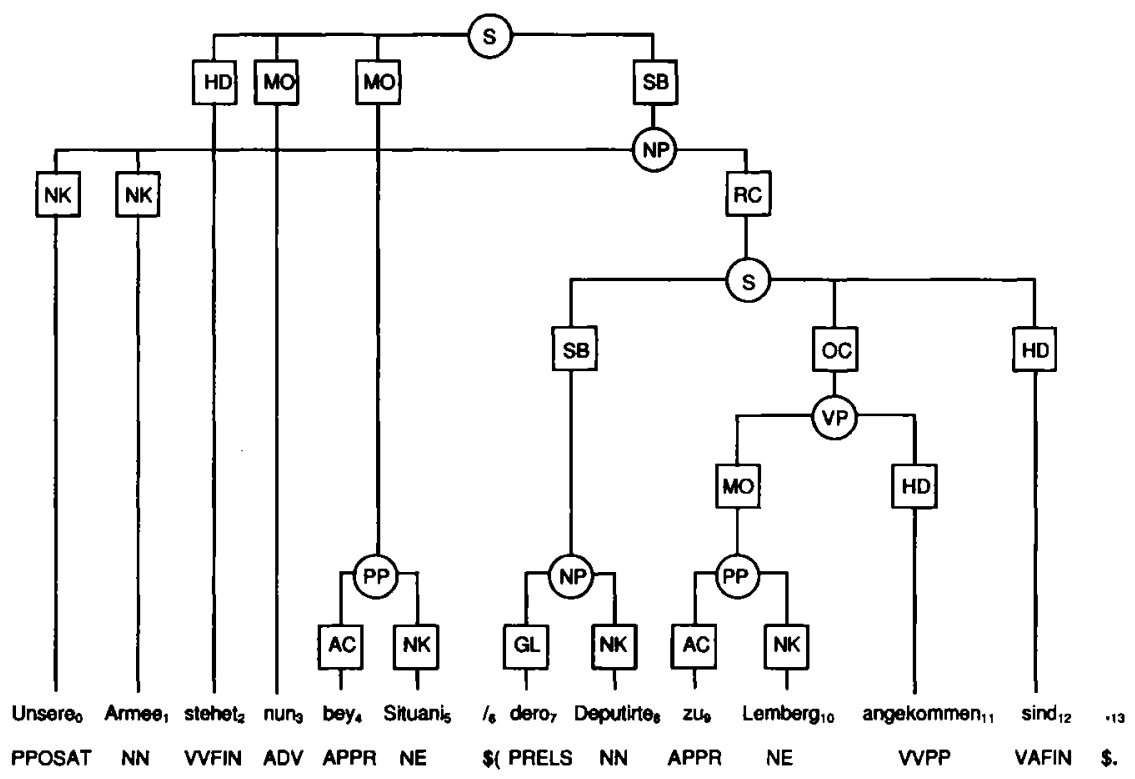

Abb. 3: Diskontinuierliche Konstituenten

Eine weitere Annotationsebene wird durch die sog. sekundären Kanten eingeführt, mit deren Hilfe die Zuordnung von Argumenten in Koordinationsstrukturen korrekt dargestellt werden kann. In einer früheren Version des Werkzeugs konnten Argumente nur mit dem nächstliegenden verbalen Kopf verknüpft werden; in der aktuellen Version können die genauen Abhängigkeitsverhältnisse durch sekundäre Kanten angezeigt werden, vgl. Abb. 4. Diese Annotationsebene ist für die Analyse der Satzstrukturen im ,Nordischen Mercurius' schon deshalb besonders interessant, weil die Dichte von komplexen Koordinationsstrukturen sehr hoch ist. Für weitere technische Details der Annotation sei auf Brants et al. (2002) verwiesen.

Die Auszeichnung erfolgt interaktiv mit Hilfe des Werkzeugs ,Annotate', einer graphischen Benutzeroberfläche, die für die Erstellung der Baumbanken ,Negra' und ,Tiger' entwickelt respektive weiterentwickelt worden ist. Interaktiv ist das Verfahren deshalb, weil auf der Grundlage von bereits annotiertem Material sukzessive Vorschläge für Wortarten, Phrasentypen und -funktionen gemacht werden, die der Annotator dann akzeptiert, modifiziert oder verwirft und einen eigenen Vorschlag zur Analyse von Wörtern oder Phrasen eingibt. Die Vorschläge für jede Annotationsebene beruhen auf statistischen Voraussagen für diese Ebene, einem Markov-Modell (Brants 


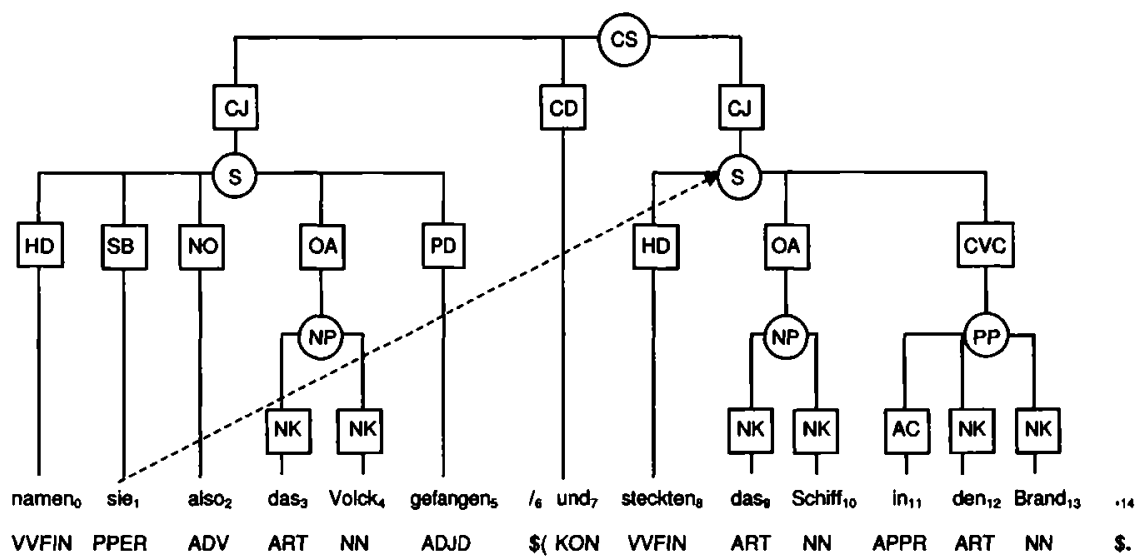

Abb. 4: Sekundäre Kanten in Koordinationsstrukturen

1999): Dem Annotator wird die Wortartkategorie und die syntaktische Phrasenstruktur (einschließlich Funktionen) mit der höchsten Wahrscheinlichkeit vorgeschlagen. Eine manuell erstellte Grammatik oder ein Lexikon werden nicht benötigt. Ganz entscheidend für die Arbeit der Annotatoren ist deshalb das regelmäßige Trainieren von Wortarttagger und Parser auf dem bereits annotierten Material, da mit der Menge der zugrunde liegenden Daten auch die Wahrscheinlichkeit wächst, dass auf Wortart- oder Phrasenebene ein richtiger Vorschlag präsentiert wird. Um die Qualität der Daten zu sichern, werden alle Sätze von zwei Annotatoren unabhängig voneinander annotiert (double keying-Verfahren) und in regelmäßigen Abständen automatisch abgeglichen. Nur durch die gemeinsame Diskussion von Problemfällen ist die Verlässlichkeit der syntaktischen Interpretation und die Optimierung des Annotationssystems zu gewährleisten.

Insgesamt enthält die Mercurius-Baumbank 30.500 Wortformen mit 8.000 Sätzen. Im Vergleich zur Tiger-Baumbank mit 900.000 Wortformen und 50.000 Sätzen ist diese erste historische Baumbank des Deutschen natürlich recht klein. Auch das syntaktisch aufbereitete Penn-Korpus des Frühneuenglischen enthält bereits 1,8 Mio. Wortformen. Aber ein Anfang für syntaktische Recherchen in historischen Texten ist gemacht.

\section{Zur Auswertung von Baumbanken}

Syntaktisch annotierte Texte enthalten eine große Menge an Informationen, die allein durch geeignete Suchwerkzeuge aus der Baumbank extrahiert werden können. Ein derartiges Suchwerkzeug ist ,TigerSearch', das in Verbindung mit der namensgleichen Baumbank entwickelt worden ist (Lezius 2002, König et al. 2003). Grundlage für ,TigerSearch' ist die Transformation der Baumbank in ein XML-Format. 


\subsection{Formulierung von Suchanfragen}

Für Suchanfragen stehen zwei Anfragesprachen zur Verfügung: (i) eine logische Abfragesprache (beruhend auf boolschen Ausdrücken und Operationen) und (ii) eine graphische Abfragesprache. Wie diese beiden Abfragesprachen funktionieren, macht man sich am Besten an einem Beispiel klar, das ausreichend komplex ist. Ein solches Beispiel wäre etwa die Suche nach Nominalphrasen, die als Teilkonstituenten einen Artikel, das Kopfnomen und einen attributiven Satz enthalten (,arity ${ }^{*}=3$ ). Zusätzliche Bedingung soll sein, dass der Attributivsatz diskontinuierlich auftritt. In der logischen Abfragesprache wäre dann wie folgt zu formulieren,

(3) NP: Artikel, Kopfnomen und Attributivsatz (diskontinuierlich)

\#n1: [cat="NP"] $>$ [cat="S"] \&

\#n1 > [pos="NN"| pos="NE"] \&

$\# \mathrm{n} 1>$ [pos="Art"] \&

$\operatorname{arity}(\# n 1,3) \&$

discontinuous(\#n1)

wobei '>' für die Dominanzrelation zwischen zwei Knoten steht und '|' alternativ einen Gattungsnamen oder einen Eigennamen als Kopf der Nominalphrase erlaubt. Suchabfragen können gespeichert werde, so dass die Ergebnisse der Suchanfrage problemlos reproduzierbar sind. In der graphischen Abfragesprache gestaltet sich die identische Anfrage so, dass Dominanz- und Präzedenzrelationen zwischen Knoten durch lineare Verbindungen einer bestimmten Art angezeigt werden:

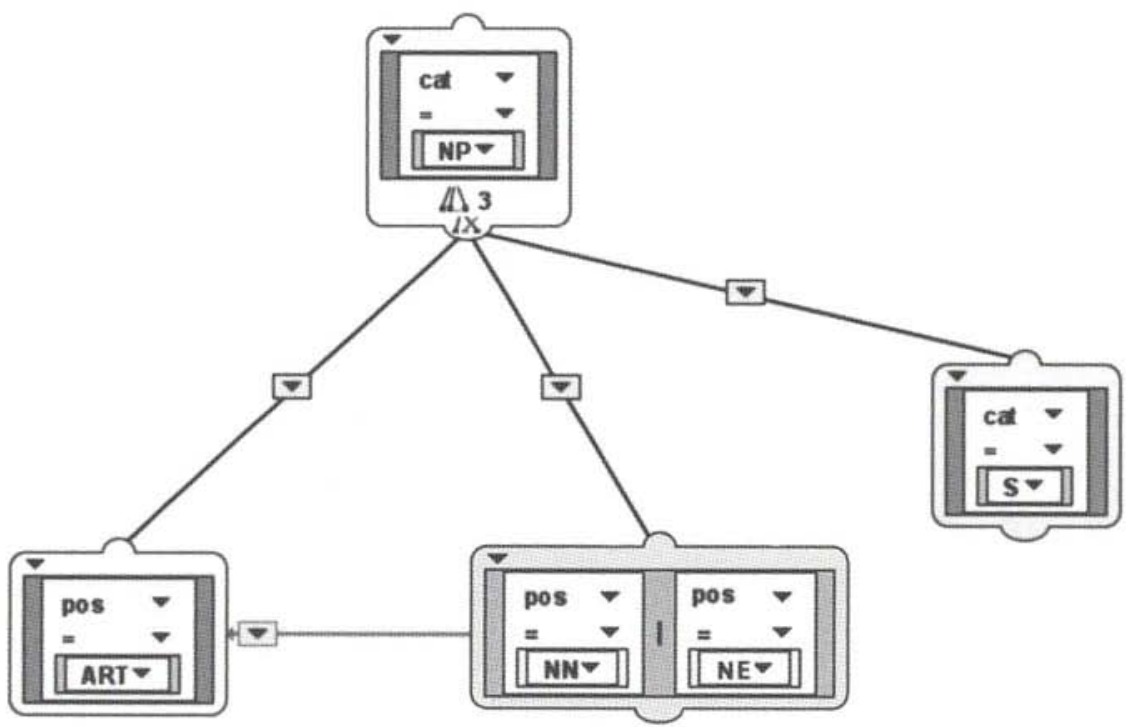

Abb. 5: Graphische Suchanfrage 
Es ist ohne weiteres möglich, graphische Suchanfragen in die logische Abfragesprache übersetzen zu lassen; umgekehrt funktioniert das allerdings nicht.

\subsection{Präsentation der Ergebnisse}

Die Ergebnisse der Suchanfrage werden unabhängig von der Abfragesprache in graphischer Form, d.h. Baumdiagrammen ausgegeben, zwischen denen hin- und hergeblättert werden kann. Die Baumdiagramme in der Ergebnisdatenbank können einzeln oder insgesamt exportiert und ausgedruckt werden. Grundsätzlich besteht bei der Darstellung der Baumdiagramme die Wahl, ob das gesamte Satzgefüge dargestellt werden soll oder ob nur der für die Suchanfrage relevante Ausschnitt gezeigt wird. Für unsere Suchanfrage ergeben sich somit zwei mögliche Darstellungen: In Abb. 6 findet sich ein Satzgefüge, in dem das gesuchte syntaktische Muster - bestehend aus einer Nominalphrase mit Artikel und diskontinuierlichem Attributivsatz - enthalten und farbig hervorgehoben ist. In Abb. 7 ist die Fundstelle reduziert auf das einschlägige syntaktische Muster, ohne dass sprachliches Material interveniert.

Die Ergebnisausgabe kann auch in Form von Tabellen geschehen, eine Form der Ausgabe, die besonders dann interessant ist, wenn Frequenzen eine Rolle spielen. Ganz abgesehen davon, dass für die Bestimmung der Produktivität eines syntaktischen Musters in einer gegebenen historischen Sprachstufe des Deutschen eine solche Abfragemöglichkeit unabdingbar ist, kann die Abfrage von Frequenzen auch dazu beitragen, die Qualität der syntaktischen Annotation zu verbessern und eine konsistente Annotation zu unterstützen. Das lässt sich etwa an der Analyse von Präpositionalphrasen demonstrieren, für die das Annotationsschema drei mögliche Funktionen vorsieht: (i) Adjunkt, (ii) Komplement und (iii) Teil eines Funktionsverbgefüges. Neben Kriterien, die für Sprecher des Gegenwartsdeutschen oft nur schwierig auf historische Texte anwendbar sind (mögliche Pronominalisierung, Erweiterbarkeit, Erfragbarkeit etc.), ist ein Kriterium wie die Reihenbildung sicher hilfreich bei der Abgrenzung der einzelnen Funktionen.

(4) a. Budoa und Castel novo nahe Ragusa sollen gantz in die See weggesuncken seyn. (M 285.21)

b. Die aus Engeland/ Franckreich und Holland melden von allen Seiten grosser Zurüstung/ ehist mit mächtigen Flooten wiederumb in die See zu kommen. (M 195.23)

Wie die nachstehende Tabelle zeigt, liegen bei den Verbindungen der Verben gehen, kommen und bringen mit der Präpositionalphrase in die See Reihenbildungen vor, während wegsinken wie die Verben laufen und begeben nur einmal in Verbindung mit der fraglichen Präpositionalphrase erscheint. Eine Analyse der Präpositionalphrase als Modifikator im ersten Fall sowie als Teil eines Funktionsverbgefüges im zweiten Fall (in dem das Verb vor allem aspektuelle und Aktionsartmerkmale trägt, etwa im Sinne von ,lossegeln'), 
kann deshalb durch eine statistische Auswertung unterstützt werden. Solche Auswertungen liefern so die Grundlage für Listen von Funktionsverbgefügen (oder von Verben mit präpositionalen Objekten), die die Arbeit der Annotatoren weiter erleichtern können.

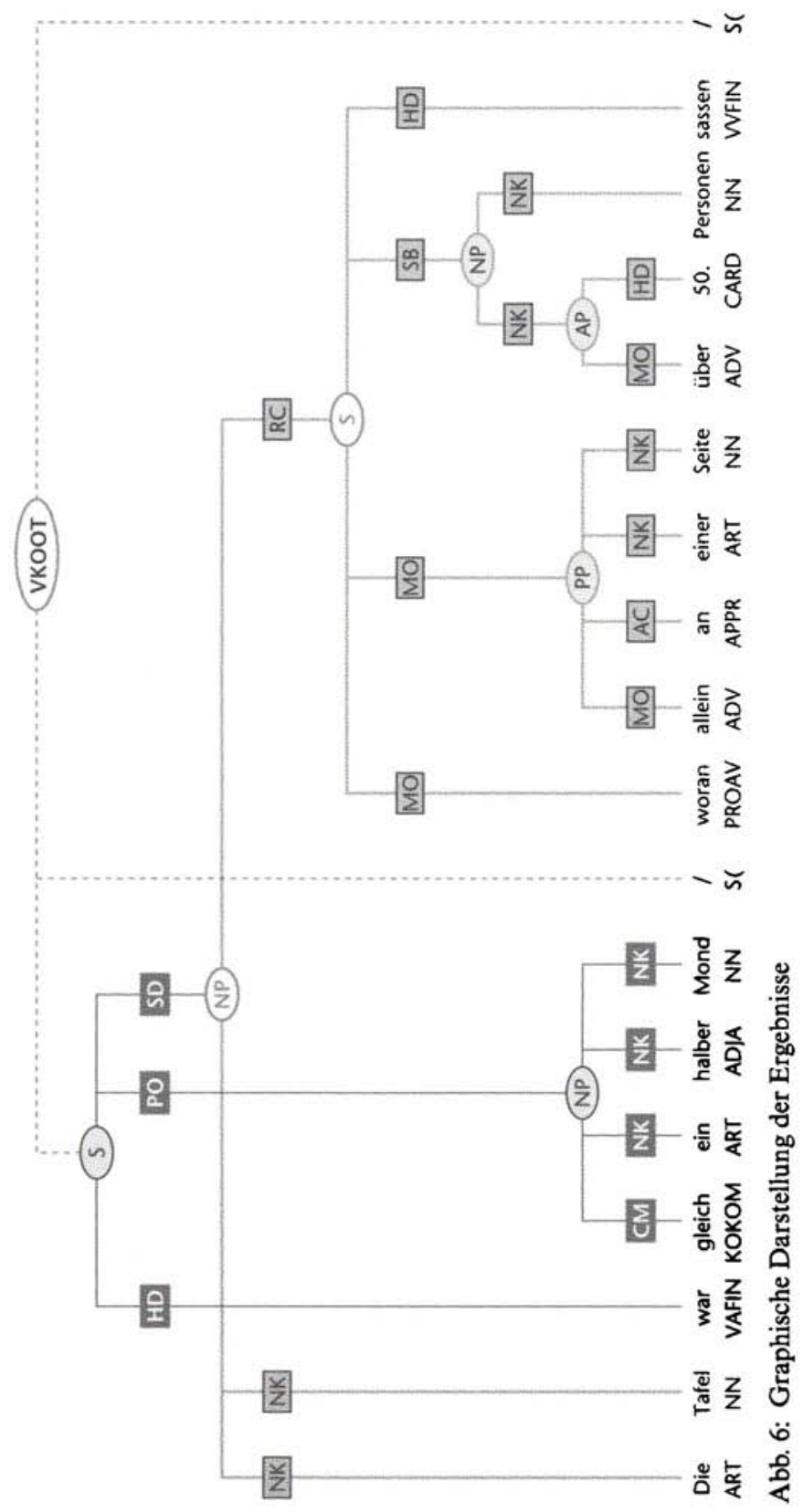




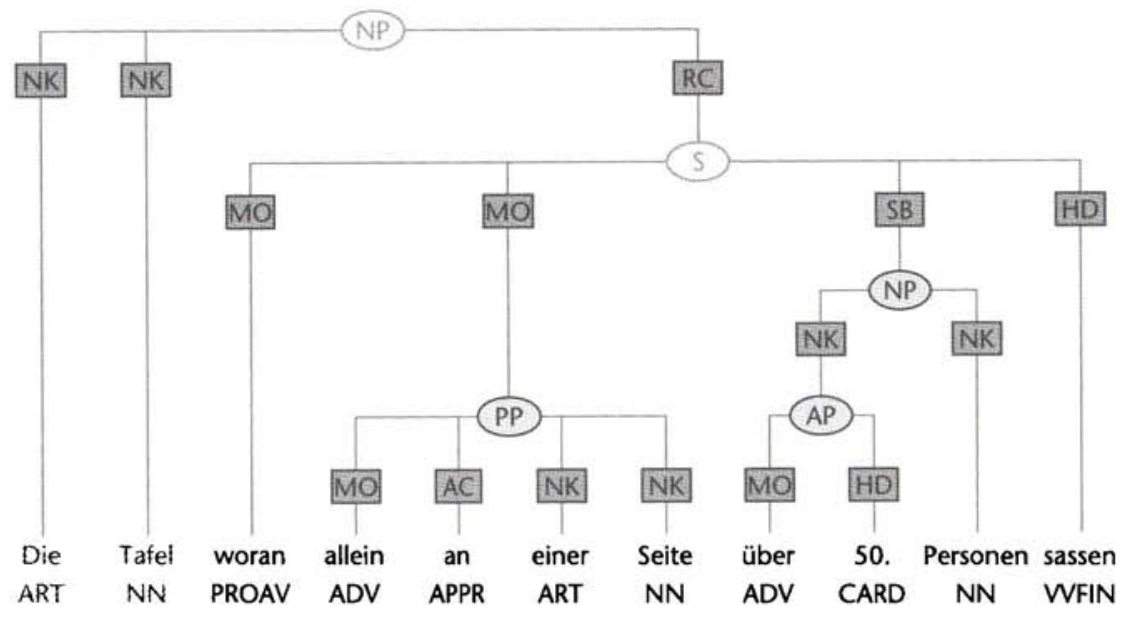

Abb. 7: Graphische Darstellung der Ergebnisse

\begin{tabular}{|l|l|l|l|l|}
\hline Frequency & Feature 1 & Feature 2 & Feature 3 & Feature 4 \\
\hline & \#t1_word & \#t2_word & \#t3_word & \#t4_word \\
\hline 10 & in & die & See & gehen \\
\hline 5 & in & die & See & kommen \\
\hline 3 & in & die & See & bringen \\
\hline 1 & in & die & See & lauffen \\
\hline 1 & in & die & See & begeben \\
\hline 1 & in & die & See & wegsinken \\
\hline
\end{tabular}

Tab. 1: Häufigkeitsverteilung der Präpositionalphrase in die See + Verb

\section{Zusammenfassung und Ausblick}

Im vorliegenden Beitrag habe ich über die Mercurius-Baumbank berichtet, ein mit syntaktischen Informationen angereichertes Zeitungskorpus aus dem späten 17. Jahrhundert. Die individuellen Ebenen der Annotation wurden beschrieben (Wortarten, Typen und Funktionen von Phrasen), Abgrenzungsprobleme von Sätzen, wie sie typischerweise in historischen Texten auftreten, thematisiert. Im letzten Abschnitt wurden schließlich die Rechercheoptionen vorgestellt, die zukünftig dazu beitragen sollen, dass in digitalisierten Texten nicht nur nach morphologischen Markern, sondern auch nach syntaktischen Mustern gesucht werden kann. Es ist offensichtlich, dass die vorliegende Baumbank die eingangs formulierten Kriterien der Repräsentativität für ein historisches Textkorpus bislang nicht erfüllt. Allerdings ist hervorzuheben, dass die Zeitungen der Frühen Neuzeit zumindest hinsichtlich der Text- 
sortenabdeckung eine angemessenere Datengrundlage liefern als andere Textquellen, weil sie eine Reihe ganz unterschiedlicher Textsorten wie Berichte, Reportagen, Dokumente, Briefe etc. umfassen. ${ }^{7}$ Dass die Textmenge, die der Mercurius-Baumbank zugrunde liegt, unbedingt der Erweiterung bedarf, um wirklich verlässliche Aussagen über die Produktivität und Veränderungen in der Produktivität syntaktischer Muster zu erhalten, versteht sich von selbst. Deshalb ist vorgesehen, baldmöglichst mit der Annotation weiterer Zeitungstexte zu beginnen. Darüber hinaus sollen die momentan abfragbaren Informationen um solche zu morphologischen Merkmalen und Lemmatisierung ergänzt werden. Auch hierzu ist bereits ein entsprechendes Werkzeug entwickelt worden (,TigerMorph'). Die Dringlichkeit dieser Forschungsaufgabe liegt auf der Hand. Das ist andernorts längst erkannt worden, weshalb beispielsweise zum Englischen entsprechende Korpora für einzelne historische Sprachstufen bereits vorliegen (Brooklyn-Geneva-Amsterdam-Helsinki Parsed Corpus of Old English, Penn-Helsinki Parsed Corpus of Middle English).

\section{Literatur}

Quellen

$[M]=$ Mercurius 1667. Nordischer Mercurius. Welcher kürtzlich vorstellet/was in diesem 1667. Jahre an Novellen aus Europa einkommen ist. Hamburg 1667.

\section{Sekundärliteratur}

Admoni, Vladimir G. (1980): Zur Ausbildung der Norm der deutschen Literatursprache im Bereich des neuhochdeutschen Satzgefüges (1470-1730). Ein Beitrag zur Geschichte des Gestaltungssystems der deutschen Sprache. Berlin. (Bausteine 56/IV).

Biber, Douglas (1993): Representativeness in corpus design. In: Literary and Linguistic Computing 8, S. 243-257.

Böning, Holger (2005): Wie man Leser glücklich macht. In: Die Zeit Nr. 25, S. 88.

Brants, Sabine/Dipper, Stefanie/Hansen, Silvia/Lezius, Wolfgang/Smith, George (2002): The TIGER Treebank. In: Proceedings of the Workshop on Treebanks and Linguistic Theories Sozopol/Bulgarien. S. 24-41.

Brants, Thorsten (1999): Tagging and Parsing with Cascaded Markov Models - Automation of Corpus Annotation. Saarbrücken: DFKI, Saarbrücken Dissertations in Computational Linguistics and Language Technology Bd. 6.

Brants, Thorsten/Skut, Wojciech/Uszkoreit, Hans (1999): Syntactic Annotation of a German Newspaper Corpus. In: Proceedings of the ATALA Treebank Workshop. Paris, S. 69-76.

Demske, Ulrike (1996): Bestandsaufnahme zum Untersuchungsbereich ,Syntax‘. In: Fritz, Gerd/Straßner, Erich (Hg.) (1996): Die Sprache der ersten deutschen Wochenzeitungen im 17. Jahrhundert. Tübingen. S. 70-125.

Demske, Ulrike/ Frank, Nicola/Laufer, Stefanie/Stiemer, Hendrik (2004): Syntactic Interpretation of an Early New High German Corpus. In: Kübler, Sandra/Nivre, Joakim/

7 Vgl. dazu vor allem Schröder (1995). 
Hinrichs, Erhard/Wunsch, Holger (eds.) (2004): Proceedings of the Third Workshop on Treebanks and Linguistics Theories (TLT 2004). Tübingen. S. 175-182.

Demske-Neumann, Ulrike (1990): Charakteristische Strukturen von Satzgefügen in den Zeitungen des 17. Jahrhunderts. In: Betten, Anne (Hg.) (1990): Neuere Forschungen zur historischen Syntax des Deutschen. Tübingen. S. 239-252.

Ebert, Robert P. (1986): Historische Syntax des Deutschen II: 1300-1750. Bern.

Gieseler, Jens/Kühnle-Xemaire, Elke (1995): Der Nordische Mercurius - eine besondere Zeitung des 17. Jahrhunderts? Eine sprachwissenschaftliche Untersuchung der Hamburger Zeitung. In: Publizistik 40, 2.

Glaser, Elvira (2003): Zu Entstehung und Charakter der neuhochdeutschen Schriftsprache: Theorie und Empirie. In: Berthele, Raphael/Christen, Helen/Germann, Sybille/Hove, Ingrid (Hg.) (2003): Die deutsche Schriftsprache und die Regionen: Entstehungsgeschichtliche Fragen in neuer Sicht. Berlin. S. 57-78.

König, Esther/Lezius, Wolfgang/Voormann, Holger (2003): TigerSearch User's Manual. University of Stuttgart.

Lezius, Wolfgang (2001): Baumbanken. In: Carstensen, Kai-Uwe/Ebert, Christian/Endriss, Cornelia/Jekat, Susanne/Klabunde, Ralf/Langer, Hagen (Hg.) (2001): Computerlinguistik und Sprachtechnologie - eine Einführung. Heidelberg. S. 377-385.

Lezius, Wolfgang (2002): Ein Suchwerkzeug für syntaktisch annotierte Textkorpora. Dissertation. Universität Stuttgart. (Arbeitspapiere des Instituts für Maschinelle Sprachverarbeitung 8,4$)$.

von Polenz, Peter (1994): Deutsche Sprachgeschichte vom Spätmittelalter bis zur Gegenwart. Bd. II: 17. und 18. Jahrhundert. Berlin.

Schröder, Thomas (1995): Die ersten Zeitungen. Textgestaltung und Nachrichtenauswahl. Tübingen.

Skut, Wojciech/Brants, Thorsten/Krenn, Brigitte/Uszkoreit, Hans (1998): A Linguistically Interpreted Corpus of German Newspaper Text. In: Proceedings of the Conference on Language Resources and Evaluation LREC-98. Granada. S. 705-711.

Smith, George/Eisenberg, Peter (2000): Kommentare zur Verwendung des STTS im NEGRA-Korpus. Manuskript. Universität Potsdam.

Solms, Hans-Joachim/Wegera, Klaus-Peter (1998): Das Bonner Frühneuhochdeutschkorpus. Rückblick und Perspektiven. In: Bergmann, Rolf (Hg.) (1998): Probleme der Textauswahl für einen elektronischen Thesaurus. Beiträge zum ersten Göttinger Arbeitsgespräch zur historischen deutschen Wortforschung am 1. und 2. November 1996. Stuttgart. S. 22-39.

Stolt, Birgit (1990): Redeglieder, Informationseinheiten: Cola und Commata in Luthers Syntax. In: Betten, Anne (Hg.) (1990): Neuere Forschungen zur historischen Syntax des Deutschen. Tübingen. S. 379-392.

Wegera, Klaus-Peter (2000): Grundlagenprobleme einer mittelhochdeutschen Grammatik. In: Besch, Werner/Betten, Anne/Reichmann, Oskar/Sonderegger, Stefan (Hg.) (2000): Sprachgeschichte. Ein Handbuch zur Geschichte der deutschen Sprache und ihrer Erforschung. Berlin. S. 1304-1320. 